\title{
External beam boost versus interstitial high-dose-rate brachytherapy boost in the adjuvant radiotherapy following breast-conserving therapy in early-stage breast cancer: a dosimetric comparison
}

\author{
Martje Marie Terheyden, MD, Corinna Melchert, PhD, Prof. György Kovács, MD, PhD \\ Interdisciplinary Brachytherapy Unit, University of Luebeck/UKSH-CL, Luebeck, Germany
}

\begin{abstract}
Purpose: This study aims to compare the dosimetric data of local tumor's bed dose escalation (boost) with photon beams (external beam radiation therapy - EBRT) versus high-dose-rate interstitial brachytherapy (HDR-BT) after breast-conserving treatment in women with early-stage breast cancer.

Material and methods: We analyzed the treatment planning data of 136 irradiated patients, treated between 2006 and 2013, who underwent breast-conserving surgery and adjuvant whole breast irradiation (WBI; 50.4 Gy) and boost (HDR-BT: $10 \mathrm{~Gy}$ in one fraction [ $n=36]$; EBRT: $10 \mathrm{~Gy}$ in five fractions $[n=100])$. Organs at risk (OAR; heart, ipsilateral lung, skin, most exposed rib segment) were delineated. Dosimetric parameters were calculated with the aid of dose-volume histograms (DVH). A non-parametric test was performed to compare the two different boost forms.

Results: There was no difference for left-sided cancers regarding the maximum dose to the heart (HDR-BT 29.8\% vs. EBRT $29.95 \%, p=0.34$ ). The maximum doses to the other OAR were significantly lower for HDR-BT (D ${ }_{\max }$ lung $47.12 \%$ vs. $87.7 \%, p<0.01$; rib $61.17 \%$ vs. $98.5 \%, p<0.01$; skin $57.1 \%$ vs. $94.75 \%, p<0.01$; in the case of right-sided breast irradiation, dose of the heart $6.00 \%$ vs. $16.75 \%, p<0.01$ ).

Conclusions: Compared to EBRT, local dose escalation with HDR-BT presented a significant dose reduction to the investigated OAR. Only left-sided irradiation showed no difference regarding the maximum dose to the heart. Reducing irradiation exposure to OAR could result in a reduction of long-term side effects. Therefore, from a dosimetric point of view, an interstitial boost complementary to WBI via EBRT seems to be more advantageous in the adjuvant radiotherapy of breast cancer.
\end{abstract}

J Contemp Brachytherapy 2016; 8, 4: 294-300 DOI: 10.5114/jcb.2016.61973

Key words: adjuvant radiotherapy, breast cancer, dosimetry, EBRT, HDR brachytherapy (HDR-BT).

\begin{abstract}
Purpose
Breast cancer is the most common form of cancer among women in the western world. The current treatment for early-stage breast cancer includes breast-conserving surgery, whole-breast irradiation, and an additional local radiation dose to the tumor bed (boost). Randomized studies have shown the benefit of boosting in order to minimize the local recurrence rate $[1,2,3,4]$. However, the radiation doses delivered to organs at risk (heart, lung, skin, rib segment) can increase long-term risks of normal tissue side effects. A recent study has shown an increase of $7.4 \%$ per Gy in the risk of ischemic heart events [5]. The risk of pneumonitis correlates with the average dose to the lung [6], and a reduction of the dose delivered to the skin shows a better cosmetic outcome $[4,7,8]$.
\end{abstract}

There are different techniques for delivering the boost: via photons, electrons, and interstitial implants. Limited data exist on the advantages of the different boost forms $[3,9]$. This study has aimed to compare the dosimetric parameters of a multicatheter single-shoot interstitial tumor-bed boost with high-dose-rate brachytherapy (HDR-BT) to those of a photon boost via external beam radiotherapy (EBRT). Data on incidental irradiation to organs at risk when boosting the tumor bed in women with early-stage breast cancer have been analyzed.

\section{Material and methods}

One hundred thirty-six patients with early-stage breast cancer who underwent breast-conserving surgery and whole breast irradiation (WBI; 50.4 Gy) between January 
2006 and December 2013 were enrolled in this retrospective investigation. Patients signed a general consent form before treatment. Table 1 summarizes patients and tumor characteristics. Thirty-six patients received an interstitial HDR-BT boost (10 Gy in 1 fraction). For a valid statistical comparison, 100 patients were randomly selected from the institutional EBRT boost group of patients (photon-boost: $10 \mathrm{~Gy}$ in 5 fractions). For the EBRT boost, a three-field three-dimensional conformal radiation therapy (3D-CRT) technique was used.

Patients at higher risk for local recurrence were recommended to receive an HDR-BT boost. If a patient refused to undergo a brachytherapy treatment, she received an EBRT boost. For each patient, the best treatment was planned individually. The target volume was defined as the former site of the tumour (clip marked and correlated to preoperative mammography images), plus a $2-3 \mathrm{~cm}$ safety margin in all directions. The HDR-BT clinical target volume encompassed the tumour bed and an at least $10 \mathrm{~mm}$ safety margin in all directions, and it was equal to the planning target volume (PTV). The minimum quality parameters were $D_{90} \geq 90 \%$ (dose delivered to $90 \%$ of the PTV), $\mathrm{D}_{\text {skin }}<50 \mathrm{~Gy}$ (maximal dose delivered to the skin), $\mathrm{V}_{150}<70 \mathrm{~cm}^{3}$ (volume receiving $150 \%$ of the reference dose). The dose non-uniformity ratio (DNR) should be < $30 \%$ [10].

\section{Contouring}

Organs at risk (heart, ipsilateral lung, skin, most exposed rib segment) were contoured on the planning computed tomography $(\mathrm{CT})$ with the treatment planning systems (BrachyVision and Eclipse/VARIAN, Palo Alto Ca, USA; OncentraBrachy/Nucletron, an Elekta company, Elekta AB, Stockholm, Sweden) according to the existing contouring atlases $[11,12]$. Dosimetric characteristics were obtained from dose-volume histograms (DVH). The DNR was calculated $(\mathrm{DNR}=\mathrm{v} 150 / \mathrm{v} 100)$ as a quality parameter of interstitial implants. The absolute volumes in cubic centimeters (cc) of the organs at risk were reported. The organ-specific description is as follows.

\section{Heart}

The whole heart was delineated with pericardium and fatty tissue. This includes the part inferior to the left pulmonary artery down to the diaphragma [11]. The dosimetric parameter was $\mathrm{D}_{\max }(\%)$ (maximum dose given in percentage), as high maximum dose values can cause atherogenic, as well as perfusion defects, and can lead to cardiovascular events [13]. Due to the reduced number of slices of the planning CT to minimize radiation exposure and the lack of contrast agents, it was not possible to delineate correctly the entire left anterior descending coronary artery (LAD) in the planning $C T$. The results of the radiation exposure to the LAD would have been flawed.

\section{Lung}

The ipsilateral lung was delineated excluding the large bronchi. The $\mathrm{D}_{\text {mean }}(\mathrm{Gy})$ and $\mathrm{D}_{\max }(\%)$ were reported for each patient. $D_{\text {mean }}(G y)$ is the mean dose in Gy to the lung - it seems to correlate best with the risk of pneumonitis $[6,14,15]$. Furthermore, the maximum dose to the lung can cause secondary malignancies $\left(\mathrm{D}_{\max }[\%]\right)$ $[16,17,18,19]$.

\section{Most exposed rib segment}

The most exposed rib segment was identified with the aid of consecutive point dose measurements on the planning CT. The maximum dose $\left(\mathrm{D}_{\max }[\%]\right)$ and the dose in Gy that irradiates $1 \mathrm{cc}$ of the rib $\left(\mathrm{d}_{1 \mathrm{cc}}\right)$ were reported.

\section{Skin}

The skin was defined as a thick layer of $5 \mathrm{~mm}$ underneath the skin surface, as, according to the data in the literature, irradiation of the vessels located in the superficial and mid-dermis can cause telangiectasia [20]. The maximum dose to the skin $\left(\mathrm{D}_{\max }[\%]\right)$ and the dose in Gy that irradiates $1 \mathrm{cc}$ of the skin $\left(\mathrm{d}_{1 \mathrm{cc}}[\mathrm{Gy}]\right)$ were analyzed for each patient.

\section{Statistical analysis}

IBM SPSS Statistics (Version 22.0.0.0, Chicago, IL, USA) was used for statistical analysis. Medians and quartiles were distinguished and non-parametric test (the Mann-Whitney-U Test) was performed on the data.

Table 1. Patient and tumor characteristics

\begin{tabular}{lcc} 
Characteristic & HDR-BT $(n=36)$ & EBRT $(n=100)$ \\
\hline Age in years & & \\
\hline Median & 58 & 62 \\
\hline Range $^{\star}$ & $54.75-65.25$ & $50-69$ \\
\hline Breast volume $[c c]$ & \\
\hline Median & 1102.6 & 1179.3 \\
\hline Range & $874-1380.75$ & $871.9-1491.2$
\end{tabular}

\begin{tabular}{lcc}
\hline Side of the tumor & \\
\hline Right-sided & 21 & 46 \\
\hline Left-sided & 15 & 54 \\
\hline Localization of tumor & & \\
\hline Upper outer quadrant & 21 & 58 \\
\hline Upper inner quadrant & 4 & 10 \\
\hline Lower outer quadrant & 2 & 17 \\
\hline Lower inner quadrant & 4 & 7 \\
\hline Other & 5 & 16 \\
\hline Size of the tumor [cm] & 18 & $11-23$ \\
\hline Median & $11-29.25$ & \\
\hline Range & & \\
\hline
\end{tabular}

$H D R-B T$ - high-dose-rate interstitial brachytherapy, EBRT - external beam radiotherapy

*Range: $25^{\text {th }}$ quartile $-75^{\text {th }}$ quartile 
The Mann-Whitney- $U$ Test was used to control for discordant values in this small database. Rank-based box plots illustrated the findings of the tests. The doses of the HDR-BT boost were compared to those of the EBRT boost. Differences in the irradiation of a right- or left-sided tumor were accessed for doses to the heart. For all other organs at risk, it was assumed that there was no difference in the doses caused by the localization of the tumor. The null hypothesis (no difference between the HDR-BT and EBRT boost irradiation) was rejected at a $p$-value less than 0.01 .

Due to different treatment schedules, the isoeffective dose can differ. We therefore calculated the biological effective dose (BED) for a better comparison between HDR-BT and EBRT. The values for the $\alpha / \beta$-ratio were chosen to be 3 Gy (heart, lung, most exposed rib), and 2.8 Gy (skin), as values for late-responding normal tissues $[21,22]$.

Table 2. Characteristics of radiotherapy

\begin{tabular}{|c|c|c|}
\hline Characteristic & HDR-BT $(n=36)$ & EBRT $(n=100)$ \\
\hline \multicolumn{3}{|c|}{ Whole breast irradiation [Gy] } \\
\hline Median dose & 50.4 & 50.4 \\
\hline Range* $^{*}$ & $50-50.4$ & $50.4-50.4$ \\
\hline \multicolumn{3}{|l|}{ Boost [Gy] } \\
\hline Median dose & 10 & 10 \\
\hline Range & $8-10$ & $10-10$ \\
\hline \multicolumn{3}{|c|}{ Target volume [cc] } \\
\hline Median & 103.61 & 131.75 \\
\hline Range & $65.54-121.66$ & $95-178.18$ \\
\hline \multicolumn{3}{|l|}{ DNR } \\
\hline Median & 0.3 & \\
\hline Range & $0.23-0.35$ & \\
\hline \multicolumn{3}{|c|}{ Number of fractions (boost) } \\
\hline Median & 1 & 5 \\
\hline Range & $1-1.5$ & $5-5$ \\
\hline \multicolumn{3}{|c|}{ Number of needles (HDR-BT) } \\
\hline Median & 15 & \\
\hline Range & $12-18$ & \\
\hline \multicolumn{3}{|c|}{ Number of planes (HDR-BT) } \\
\hline Median & 3 & \\
\hline Range & $3-4$ & \\
\hline \multicolumn{3}{|c|}{ Number of fields (EBRT) } \\
\hline Median & & 3 \\
\hline Range & & $3-3$ \\
\hline
\end{tabular}

\section{Results}

There were 36 eligible interstitial HDR-BT cases, from which 21 patients received right-sided irradiation and 15 patients left-sided irradiation. In the EBRT group, there were 46 right-sided and 54 left-sided tumors. Patient and tumor characteristics are given in Table 1.

The reference dose-line was the $100 \%$ dose-line on the planning CTs. The results are listed as medians. The range indicates values in the $25^{\text {th }}$ quartile and the $75^{\text {th }}$ quartile, given in brackets.

The DNR, as a parameter for quality and a prognostic factor for acceptable toxicity, for interstitial implants should be below 0.35 [23]. In this study, the mean DNR was $0.3(0.23-0.35)$, which shows an excellent result and well-calculated interstitial HDR-BT plans. The characteristics of radiotherapy are given in Table 2.

\section{Heart}

Tumors located on the left side led to a higher maximum dose of irradiation to the heart than tumors located on the right side: HDR-BT left-sided irradiation $29.8 \%(19.58-32.68 \%)$ vs. right-sided irradiation $6.00 \%$ (4.79-8.07\%); left-sided EBRT 29.95\% (21.80-49.98\%) vs. right-sided EBRT 16.75\% (12.67-22.28\%).

Our study has shown no significant difference between the two local dose escalation techniques in the heart dose reduction regarding left-sided tumors $(p \approx 0.34)$. Radiation of right-sided cancer with interstitial implants showed less radiation exposure to the heart compared to EBRT ( $p<0.01$; Figure 1).

The mean dose to the heart was 0.27 Gy (0.19-0.59 Gy) for HDR-BT patients. In patients with left-sided breast cancer, the heart received 0.67 Gy (0.39-1.80 Gy); the mean heart dose of patients with right-sided irradiation was 0.24 Gy $(0.17-0.27 \mathrm{~Gy})$.

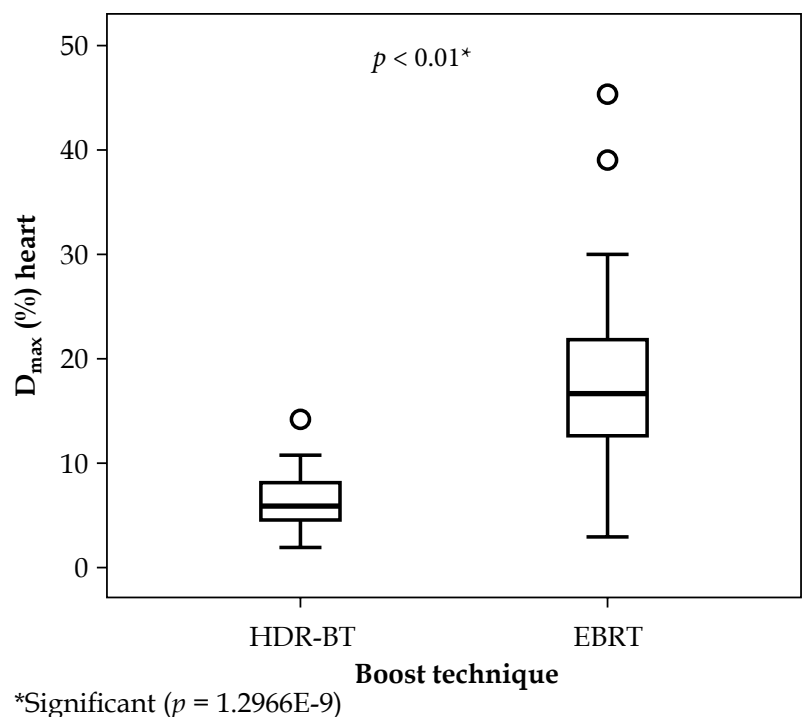

Fig. 1. Box plot of irradiation of the heart (right-sided breast cancer): there is a significant difference between external beam radiotherapy (EBRT) and high-dose-rate interstitial brachytherapy (HDR-BT) 
For EBRT patients, the mean heart dose was $0.37 \mathrm{~Gy}$ (0.16-0.68 Gy). Regarding the left-sided irradiation, the heart received 0.50 Gy (0.29-0.86 Gy) vs. 0.23 Gy (0.1-0.47 Gy) for a right-sided tumor.

\section{Lung}

The ipsilateral lung volume of the interstitial irradiated patients was $1245.95 \mathrm{cc}$, while, for the EBRT patients it was 1235.35 cc.

With HDR-BT, the lung received maximum doses of 3.25 Gy (1.99-4.68 Gy), and with EBRT, 8.74 Gy (7.42$9.34 \mathrm{~Gy})$. The difference is significant ( $p<0.01$, Figure 2).

Mean doses to the lung are understood as markers for pneumonitis risk. A higher mean dose indicates higher pneumonitis risk. Our data show a significant reduction of the mean dose with interstitial implants: $0.40 \mathrm{~Gy}(0.27-$ $0.57 \mathrm{~Gy})$ vs. 0.79 Gy $(0.52-0.99 \mathrm{~Gy}), p<0.01$.

\section{Most exposed rib segment}

The volume of the most exposed rib segment was $19.25 \mathrm{cc}$ (HDR-BT) vs. $22.8 \mathrm{cc}$ (EBRT). The maximum dose was calculated at $4.29 \mathrm{~Gy}$ (2.76-6.27 Gy) for HDR-BT and 9.84 Gy (9.55-10.05 Gy) for EBRT.

The maximum dose $\left(\mathrm{D}_{\max }[\%]\right)$ of EBRT given in the DVH is significantly higher than the dose of interstitial implants: $98.5 \%$ (96.11-100.4\%) vs. $61.17 \%$ (41.24-77.64\%), $p<0.01$ (Figure 3). Additionally, $1 \mathrm{cc}$ of rib received less radiation with an HDR-BT boost: 3.37 Gy (1.85-4.59 Gy) vs. 9.27 Gy (8.52-9.70 Gy), $p<0.01$.

\section{Skin}

The HDR-BT technique reduces the maximum dose to the skin compared to EBRT. The maximum dose was calculated to be 4.01 Gy (2.28-6.72 Gy) with HDR-BT and 9.44 Gy (8.54-9.92 Gy) with EBRT. The same effect is visible when comparing the percentage maximum

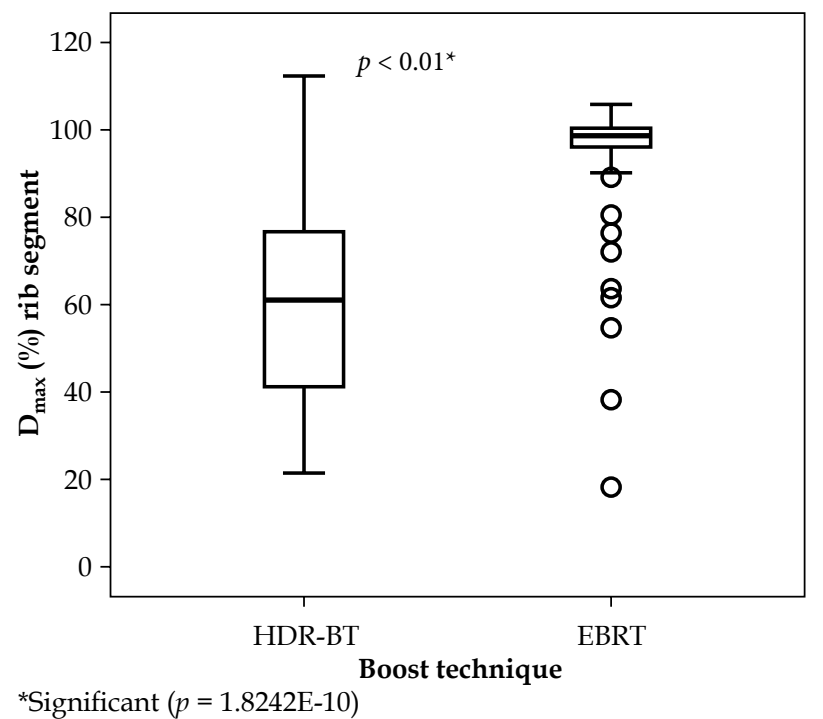

Fig. 3. Box plot of irradiation of the most exposed rib segment. There is a significant difference between the two treatment modalities

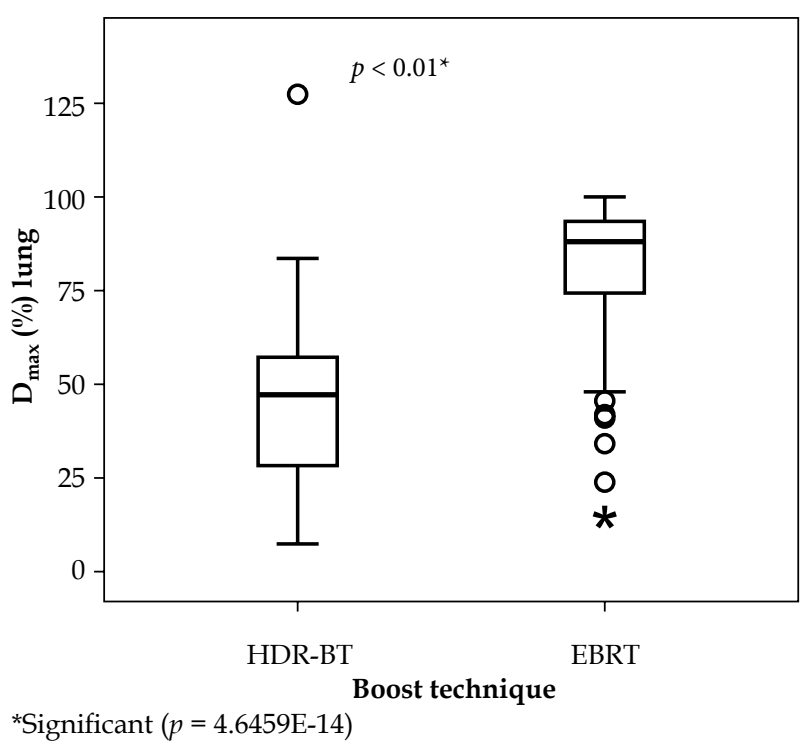

Fig. 2. Box plot of irradiation of the lung. It shows a significant difference between the two techniques in favour of high-dose-rate interstitial brachytherapy (HDR-BT)

doses: HDR-BT 57.1\% (41.71-81.15\%) vs. EBRT 94.75\% $(86.17-98.94 \%) ; p<0.01$ (Figure 4 ). One cubic centimeters of skin is significantly less irradiated with the HDR-BT boost than with EBRT: 3.23 Gy (1.86-4.90 Gy) vs. 8.57 Gy (7.59-9.28 Gy); $p<0.01$.

\section{Biological effective dose}

There are differences in the treatment regimens between the HDR-BT and EBRT. Even after adjusting the doses using the BED equation, OAR received significantly less radiation with HDR-BT. Table 3 lists the adjusted BED values.

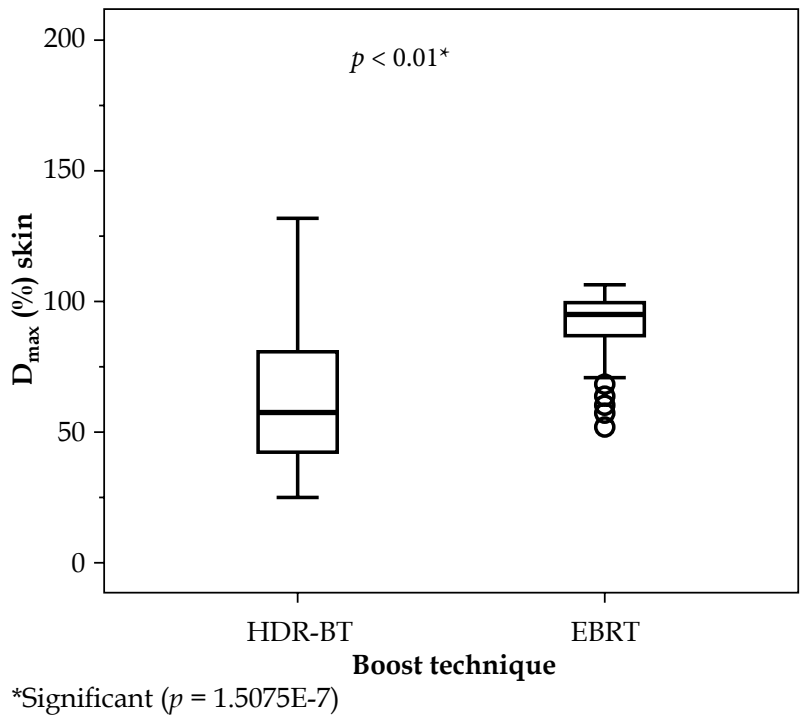

Fig. 4. Box plot of irradiation of the skin. There is a significant difference between the two treatment modalities 
Table 3. Biological effective dose (BED) values for heart, lung, most exposed rib segment, and skin

\begin{tabular}{lccc} 
& HDR-BT [Gy] & EBRT [Gy] & $p$-value \\
\hline Heart $D_{\max }$ & $5.37(4.00-7.76)$ & $10.73(8.49-16.36)$ & $<0.01$ \\
\hline Heart $D_{\max }$ left-sided irradiation $(\alpha / \beta=3)$ & $8.83(5.90-13.30)$ & $14.47(10.4-21.37)$ & $<0.01$ \\
\hline Heart $D_{\max }$ right-sided irradiation $(\alpha / \beta=3)$ & $4.29(3.85-5.20)$ & $9.27(7.64-10.94)$ & $<0.01$ \\
\hline Lung $D_{\max }(\alpha / \beta=3)$ & $12.61(8.70-17.00)$ & $32.47(27.64-34.46)$ & $<0.01$ \\
\hline Lung $D_{\operatorname{mean}}(\alpha / \beta=3)$ & $4.24(3.80-5.10)$ & $5.92(5.05-6.63)$ & $<0.01$ \\
\hline Rib $D_{\max }(\alpha / \beta=3)$ & $15.92(10.80-21.52)$ & $36.12(35.18-36.83)$ & $<0.01$ \\
\hline Skin $D_{\max }(\alpha / \beta=2.8)$ & $16.20(11.37-27.17)$ & $37.27(33.36-39.01)$ & $<0.01$
\end{tabular}

$H D R-B T$ - high-dose-rate interstitial brachytherapy, EBRT - external beam radiotherapy, $D_{\text {max }}$ - maximum dose [Gy], $D_{\text {mean }}-$ mean dose [Gy] Range: $25^{\text {th }}$ quartile $-75^{\text {th }}$ quartile

\section{Discussion}

There are only a few published studies that compare dosimetric characteristics between the different local dose escalation modalities. Our study has been able to record characteristics of maximum doses to the heart, and thus provide insight into the evaluated forms of irradiation. High-dose-rate brachytherapy plans for right-sided breast cancer have shown a reduction of the maximum doses to the heart. Nonetheless, it is important to keep in mind that the maximum doses to the heart depend on the anatomy of the chest and on the inter-/intra-fraction movements of the heart $[24,25,26,27]$.

In 2013, a Korean study compared electron and X-ray beams for tumor bed boost irradiation in 131 patients with left-sided breast cancer [28]. The maximum dose to the left ventricle was $3.66 \pm 2.70$ Gy (average \pm standard deviation). We calculated the maximum dose of the photon boost to be $3.34 \mathrm{~Gy}$, which lies within the same range as the results of Park and Kim. In our study, the HDR-BT boost reduced the maximum doses to the heart (maximum dose $1.65 \mathrm{~Gy}$ ).

In regard to the treatment of a left-sided tumor, there were no significant differences between the two techniques in terms of $\mathrm{D}_{\max }(\%)$. One limitation of the evaluation presented is that we did not consider the localization or depth of the tumor bed. Tumors located deeper in the breast are closer to the heart and lead to a higher maximum dose to the heart. It is possible that our study could have analyzed more deeply-located tumors, and thus produced erroneously high results.

The DVH contains no information on the exact localization of the maximum dose. It makes a difference if muscle or coronary arteries are irradiated. Irradiation of vessels can lead to arteriosclerosis and can cause a myocardial infarction [13].

A study on pneumonitis risk due to irradiation of breast cancer has shown a reduction of the risk by reducing the high dose volume of the lung [29]. The pneumonitis risk is proportional to the mean dose to the lung $[6,14,15,29]$.

In the study presented, the HDR-BT boost showed a reduction of the mean dose and of the maximum dose to the lung compared to the EBRT boost (mean dose 0.40 Gy vs. 0.79 Gy; maximum dose $47.12 \%$ vs. $87.7 \%$ ). Van Parijs published average dose values for the ipsilateral lung between 0.47 Gy and 1.09 Gy [30]. Our study has been able to confirm the reported doses by Van Parijs.

Another long-term risk is the development of secondary malignancies after irradiation. Women who received radiation therapy as part of their breast cancer treatment are at higher risk for secondary malignancies of the lung after 15 years [17]. Grantzau reported that the risk for lung cancer after irradiation increases linearly with every Gray delivered to the lung, by $8.3 \%$. The risk for smokers is even higher (17.3\% per Gy) [18]. The reduction of the maximum lung dose decreases the risk of secondary cancer. According to our results, the HDR-BT boost seems to deliver fewer maximum doses to the lung. Randomized studies with larger cohorts are needed to confirm these findings.

Only limited data exist on the dosimetric characteristics of irradiated ribs. Long-term risks to ribs have scarcely been documented. There is one study that published dosimetric data on the rib with a HDR-BT boost [31]. In that study, the most exposed rib received $24.5 \%$ of the prescribed dose. Potentially, the variation in the depth of the tumor could explain the different results in their study as compared to our study. More studies on the doses delivered to the rib are necessary to obtain valid data.

Many publications have shown that minimizing the dose to the skin leads to a better cosmetic outcome $[4,7$, $28,32,33,34,35]$.

Our results show a dose reduction with the HDRBT boost. When evaluating the results, one has to keep in mind that the outcomes depend on the localization and the depth of the tumor. The more superficial the tumor, the higher the maximum dose to the skin. It is also important to consider the skin-delineation technique for treatment planning. The detected doses to the skin change when defining a different width of the skin [24].

At the moment, there is no consistent recommendation regarding skin delineation for radiation treatment planning [26,36]. It is difficult to compare our findings for the maximum doses delivered to the skin to other results. Without knowing the exact localization of the tumor, it would also not be valid to compare the results. 


\section{Limitations}

The retrospective design is a further limitation of our investigation. Women were not randomized equally for either the HDR-BT boost or EBRT boost. A retrospective design cannot control bias, because of different prognostic factors. Additionally, it is important to stress the individual anatomical differences between women. It is disputable whether or not the women can be generally compared. Another limitation is the missing documentation of the exact localization and depth of the tumor.

Our presented cohort contains only a low number of cases - a higher number of cases would lead to more reliable results. Furthermore, the study only examined the dosimetric characteristics, but not the clinical results of the irradiation. Last but not least, we have neither assessed the recurrence rate nor the cosmetic outcome after treatment. Further studies with larger cohorts and longterm follow-up are necessary in the future.

Another limitation is the fact that we only examined 3D-planned photon boost cases in the EBRT group. We excluded data on electron boosts for technical reasons. Prospective studies on the comparison between electron, photon, and interstitial boosts are needed.

Studies have also shown that the contouring and the delineation processes depend on the examiner. Hence, the results can vary widely $[37,38]$. An advantage of this presentation is that the same person performed delineation on all the subjects, and the contouring atlases were considered.

Multiple new irradiation techniques are emerging (intraoperative radiotherapy - IORT), intensity-modulated radiotherapy (IMRT), irradiation in prone position, deep inspiration breath-hold techniques (DIBH), and need to be assessed. Long-term data are still lacking, but early findings have shown a benefit of these methods $[39,40]$. More studies with prospectively collected data are now in progress and need to be evaluated in the future.

\section{Conclusions}

In this limited retrospective study, we were able to demonstrate a reduction of doses delivered to organs at risk with the use of an HDR-BT boost, following WBI via EBRT in breast-conserving treatments in patients with early-stage breast cancer. We have provided data on dosimetric characteristics, and stress the importance of reducing the delivered dose to organs at risk in order to minimize long-term side effects.

\section{Disclosure}

Authors report no conflict of interest.

\section{References}

1. Bartelink H, Horiot JC, Poortmans PM et al. Recurrence rates after treatment of breast cancer with standard radiotherapy with or without additional radiation. N Engl J Med 2001; 345: 1378-1387.

2. Bartelink H, Horiot JC, Poortmans PM et al. Impact of a higher radiation dose on local control and survival in breast-cancer: 10-year results of the randomized boost versus no boost EORTC 22881-10882 trial. J Clin Oncol 2007; 25: 3259-3265.
3. Polgár C, Fodor J, Orosz Z et al. Electron and high-dose-rate brachytherapy boost in the conservative treatment of stage I-II breast cancer: first results of the randomized Budapest boost trial. Strahlenther Onkol 2002; 178: 615-623.

4. Poortmans PM, Colette L, Horiot JC et al. Impact of the boost dose of 10 Gy versus 26 Gy in patients with early stage breast cancer after a microscopically incomplete lumpectomy: 10-year results of the randomized EORTC boost trial. Radiother Oncol 2009; 90: 80-85.

5. Darby SC, Ewertz M, McGale P et al. Risk of ischemic heart disease in women after radiotherapy for breast cancer. $N$ Engl J Med 2013; 368: 987-998.

6. Seppenwoolde Y, Lebesque JV, de Jaeger K et al. Comparing different NTCP models that predict the incidence of radiation pneumonitis. Normal tissue complication probability. Int J Radiat Oncol Biol Phys 2003; 55: 724-735.

7. Collette S, Collette L, Budiharto T et al. Predictors of the risk of fibrosis at 10 years after breast conserving therapy for early breast cancer: a study based on the EORTC trial 2281-10882 "boost versus no boost". Eur J Cancer 2008; 44: 2587-2599.

8. Taylor ME, Perez CA, Halverson KJ et al. Factors influencing cosmetic results after conservation therapy for breast cancer. Int J Radiat Oncol Biol Phys 1995; 31: 753-764.

9. Poortmans P, Bartelink H, Horiot JC et al. The influence of the boost technique on local control in breast conserving treatment in the EORTC "boost versus no boost" randomized trial. Radiother Oncol 2004; 72: 25-33.

10. Strnad V, Pötter R, Kovács G. Practical Handbook of Brachytherapy. UNI-MED, Bremen 2014; pp. 184-189.

11. Feng M, Moran JM, Koelling T et al. Development and validation of a heart atlas to study cardiac exposure to radiation following treatment for breast cancer. Int J Radiat Oncol Biol Phys 2011; 79: 10-18.

12. Kong FM, Ritter T, Quint DJ et al. Consideration of dose limits for organs at risk of thoracic radiotherapy: atlas for lung, proximal bronchial tree, esophagus, spinal cord, ribs, and brachial plexus. Int J Radiat Oncol Biol Phys 2011; 81: 1442-1457.

13. Lee MS, Finch W, Mahmud E. Cardiovascular complications of radiotherapy. Am J Cardiol 2013; 112: 1688-1696.

14. Kwa SLS, Lebesque JV, Theuws JCM et al. Radiation pneumonitis as a function of mean lung dose: an analysis of pooled data of 540 patients. Int J Radiat Oncol Biol Phys 1998; 42: 1-9.

15. Kahán Z, Csenki M, Varga Z et al. The risk of early and late lung sequelae after conformal radiotherapy in breast cancer patients. Int J Radiat Oncol Biol Phys 2007; 68: 673-681.

16. Deutsch M, Land SR, Begovic M et al. The Incidence of Lung Carcinoma after Surgery for Breast Carcinoma with and without Postoperative Radiotherapy. Results of National Surgical Adjuvant Breast and Bowel Project (NSABP) Clinical Trials B-04 and B-06. Cancer 2003; 98: 1362-1368.

17. Grantzau T, Mellemkjær L, Overgaard J. Second primary cancers after adjuvant radiotherapy in early breast cancer patients: A national population based study under the Danish Breast Cancer Cooperative Group (DBCG). Radiother Oncol 2013; 106: 42-49.

18. Grantzau T, Thomsen MS, Vaeth M et al. Risk of second primary lung cancer in women after radiotherapy for breast cancer. Radiother Oncol 2014; 111: 366-373.

19. Roychoudhuri R, Evans H, Robinson D et al. Radiation-induced malignancies following radiotherapy for breast cancer. Br J Cancer 2004; 91: 868-872.

20. Van Limbergen E, Briot E, Drijkoningen M. The source-skin distance measuring bridge: A method to avoid radiation telangiectasia in the skin after interstitial therapy for breast cancer. Int J Radiat Oncol Biol Phys 1990; 18: 1239-1244. 
21. Joiner M, Kogel A. Basic clinical radiobiology. Hodder Arnold, London 2009; pp. 102-134.

22. Fowler JF. 21 years of Biologically Effective Dose. Br J Radiol 2010; 554-568.

23. Dutta S, Sharma SC, Sigh R et al. Quality Assessment of Interstitial Implants in High-Dose-Rate Brachytherapy After Lumpectomy in Patients of Early Stage Breast Cancer. Indian J Surg Oncol 2010; 1: 294-302.

24. Berger D, Kauer-Dorner D, Seitz W et al. Concepts for critical organ dosimetry in three-dimensional image-based breast brachytherapy. Brachytherapy 2008; 7: 320-326.

25. Duma MN, Molls M, Trott KR. From heart to heart for breast cancer patients - cardiovascular toxicities in breast cancer radiotherapy. Strahlenther Onkol 2014; 190: 5-7.

26. Major T , Polgár C, Lövey $\mathrm{K}$ et al. Dosimetric characteristics of accelerated partial breast irradiation with $\mathrm{CT}$ image-based multicatheter interstitial brachytherapy: A single institution's experience. Brachytherapy 2011; 10: 421-426.

27. Taylor CW, Nisbet A, McGale P et al. Cardiac exposures in breast cancer radiotherapy: 1950s-1990s. Int J Radiat Oncol Biol Phys 2007; 69: 1484-1495.

28. Park SH, Kim JC. Comparison of electron an x-ray beams for tumour bed boost irradiation in breast-conserving treatment. J Breast Cancer 2013; 16: 300-307.

29. Willner J, Jost A, Baier K et al. A Little to a Lot or a Lot to a Little? An Analysis of Pneumonitis Risk from Dose-Volume Histogram Parameters of the Lung in Patients with Lung Cancer Treated with 3-D Conformal Radiotherapy. Strahlenther Onkol 2003; 179: 548-556.

30. Van Parijs H, Reynders T, Heuninckx K et al. Breast conserving treatment for breast cancer: dosimetric comparison of different non-invasive techniques for additional boost delivery. Radiat Oncol 2014; 9: 36.

31. Wiercinska J, Wronczewska A, Kabacinska R et al. Transition from Paris dosimetry system to 3D image-guided planning in interstitial breast brachytherapy. J Contemp Brachytherapy 2015; 6: 479-484.

32. Dodwell DJ, Povall J, Gerrard G et al. Skin teleangiectasia: the influence of radiation dose delivery parameters in the conservative management of early breast cancer. Clin Oncol 1995; 7: 248-250.

33. Ott OJ, Lotter M, Sauer R et al. Accelerated partial-breast irradiation with interstitial implants - the clinical relevance of the calculation of skin doses. Strahlenther Onkol 2007; 183: 426-431.

34. Perera F, Chisela F, Stitt L et al. TLD skin dose measurements and acute and late effects after lumpectomy and high-doserate brachytherapy only for early breast cancer. Int J Radiat Oncol Biol Phys 2005; 62: 1283-1290.

35. Vrieling C, Collette L, Fourquet A et al. The influence of the boost in breast-conserving therapy on cosmetic outcome in the EORTC "boost versus no boost" trial. Int J Radiat Oncol Biol Phys 1999; 45: 677-685.

36. Lasota J, Kabacinska R, Makarewicz R. Dose estimation for different skin models in interstitial breast brachytherapy. J Contemp Brachytherapy 2014; 6: 200-207.

37. Hurkmans CW, Borger JH, Pieters BR et al. Variability in target volume delineation on CT scans of the breast. Int J Radiat Oncol Biol Phys 2001; 50: 1366-1372.

38. Li XA, Tai A, Arthur DW et al. Variability of target and normal structure delineation for breast cancer radiotherapy: an RTOG multi-institutional and multiobserver study. Int J Radiat Oncol Biol Phys 2009; 73: 944-951.

39. Bruzzaniti V, Abate A, Pinnarò O et al. Dosimetric and clinical advantages of deep inspiration breath-hold (DIBH) during radiotherapy of breast cancer. J Exp Clin Cancer Res 2013; 32: 88.

40. Varga Z, Cserháti A, Rárosi F et al. Individualized positioning for maximum heart protection during breast irradiation. Acta Oncol 2014; 53: 58-64. 